\title{
Modified posterior approach to the hip joint
}

\section{Iyer Krishna Mohan*}

Formerly Locum Consultant Orthopaedic Surgeon, Royal Free Hampstead NHS Trust, Royal Free Hospital, London, United Kingdom

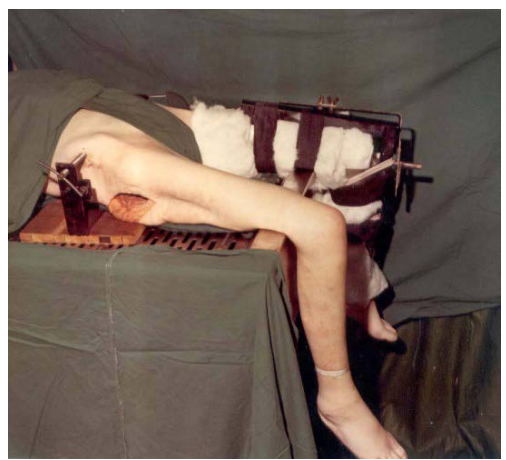

Image 1. Device used to test stability of the Hip Joint showing the pelvis fixed and protractors to measure the angle offlexion/extension, adduction/abduction and internal rotation/external rotations (Courtesy: Photographreproduced with the kind permission of Injury/ Elsevier).

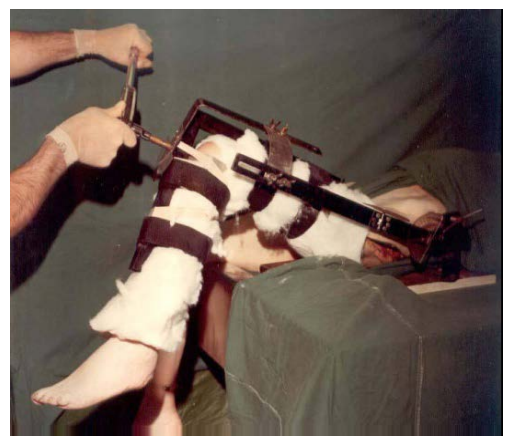

Image 2. Device used to test stability of the Hip Joint showing the pelvis fixed and protractors to measure the angle of flexion/ extension,adduction/abduction and internal rotation/external srotations (Courtesy: Photographreproduced with the kind permission of Injury/Elsevier).

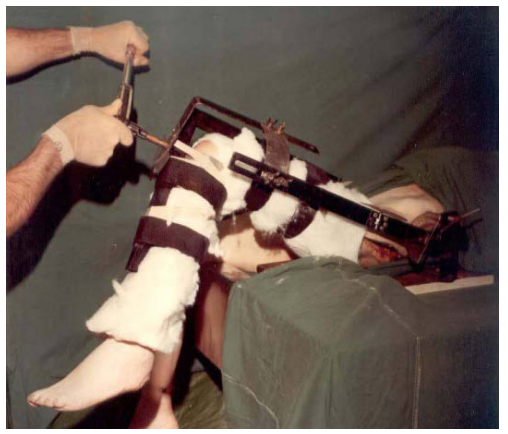

Image 3. Internal rotation torque being applied when the Hip Joint is standardized to a fixed angle of flexion and adduction(Courtesy: Photographreproduced with the kind permission of Injury/Elsevier).

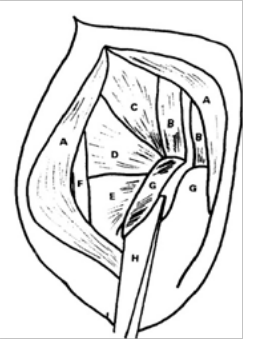

Image 4: Line Diagram showing the osteotomy of the posterior overhanging part of the greater trochanter: (Courtesy: Line Diagram reproduced with the kind permission of Injury/Elsevier): A: Gluteus maximus; B: Gluteus medius; C: Piriformis; D: Triradiate tendon; E: Quadratusfemoris; F: Sciatic nerve; G: Greater trochanter, H: Osteotome.

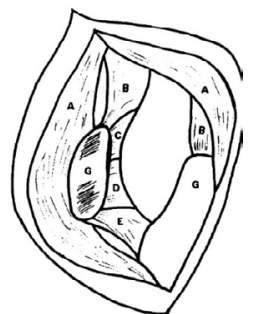

Image 5. Line Diagram showing the osteotomy completed and the flap retracted. (Courtesy:Line Diagram reproduced with the kind permission of Injury/Elsevier);A: Gluteus maximus; B: Gluteus medius; C: Piriformis; D: Triradiate tendon; E: Quadratusfemoris; G: Greater trochanter.

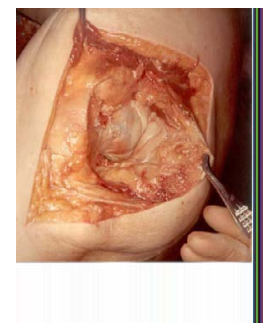

Image 6. Line Diagram to show that the Osteotomy is completed and the flap retracted,after incising the capsule to expose the Hip Joint, (Courtesy: Reproduced with the kind permission of Injury/Elsevier).

Correspondence to: Iyer Krishna Mohan, Formerly Locum Consultant Orthopaedic Surgeon, Royal Free Hampstead NHS Trust, Royal Free Hospital, London, United Kingdom, E-mail: kmiyer28@hotmail.com

Received: October 15, 2016; Accepted: November 01, 2016; Published: November 03, 2016 
Line diagram showing the following structures: A: Gluteus maximus; B: Gluteus medius; C: Piriformis; D: Triradiate tendon; E: Quadratusfemoris; G: Greater trochanter; I: Acetabulum; J: Femoral head.

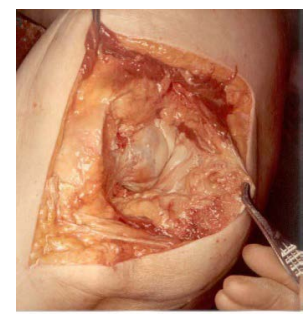

Image 7. Hip Joint opened (Courtesy: Photographreproduced with the kind permission of Injury/Elsevier).

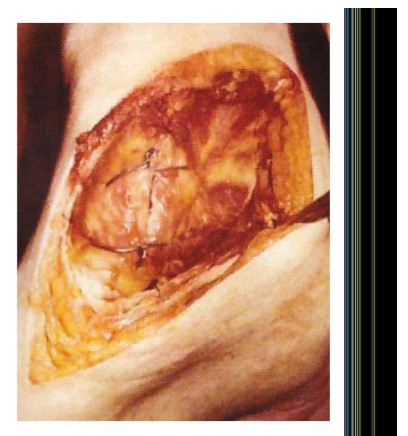

Image 8. Wiring of the trochanteric fragment (Courtesy:Photographreproduced with the kind spermission of Injury/ Elsevier).

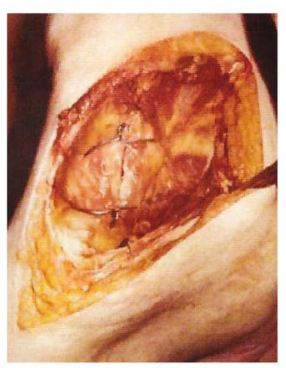

Image 9. Hip Joint reconstituted (Courtesy: Photographreproduced with the kind permission ofInjury/Elsevier).

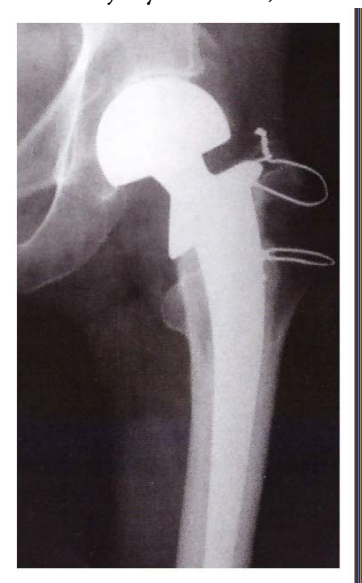

Image 10. Radiograph of total Hip prosthesis(Courtesy:Radiograp hreproduced with the kind permission of Injury/Elsevier).

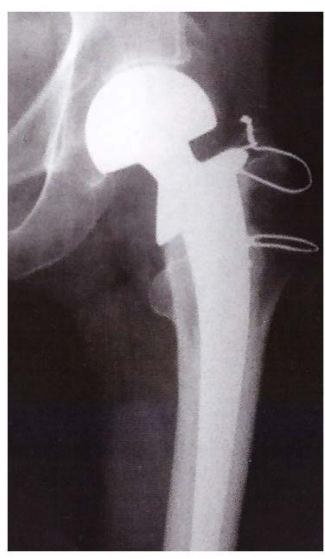

Image 11. Radiograph of Bipolar prosthesis (Courtesy: Radiographreproduced with the kind permission of Injury/Elsevier).

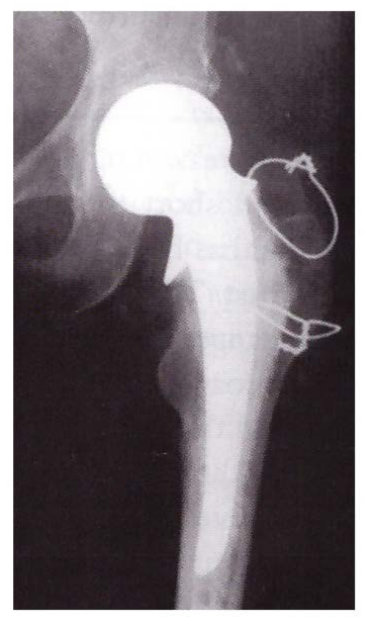

Image 12. Radiograph of Thompson's hemi-arthroplasty (Courtesy: Radiographreproduced with the kindspermission ofInjury/Elsevier).

Copyright: (C2016 Mohan IK. This is an open-access article distributed under the terms of the Creative Commons Attribution License, which permits unrestricted use, distribution, and reproduction in any medium, provided the original author and source are credited. 